\title{
Modular chaos for random processes
}

\author{
Marat Akhmet* \\ Department of Mathematics, Middle East Technical University, 06800 Ankara, Turkey
}

\begin{abstract}
In the present paper, an essential generalization of the symbolic dynamics is considered. We apply the notions of abstract self-similar sets and the similarity map for a chaos introduction, which orbits are expanded among infinitely many modules. The dynamics is free of dimensional, metrical and topological assumptions. It unites all the three types of Poincaré, Li-Yorke and Devaney chaos in a single model, which can be unbounded. The research demonstrates that the dynamics of Poincaré chaos is of exceptional use to analyze discrete and continuous-time random processes. Examples, illustrating the results are provided.
\end{abstract}

\section{Introduction}

The paradigm of chaos relies on the two distance antagonists, proximality and separation. They utilize metrical or topological closeness and divergence in the description of chaos considering either couples of orbits [1,2] or a single motion [3. In the present research, we have renounced the idea that transitivity and density of a class of motions have to be described metrically, and even topologically. Our suggestions are based on the new idea that closeness is considered not in the metrical sense, but through indexing, although separation still rely on the distance. That is, the antagonists still are present, but not in the way of conventional theories. The process of indexing is adjacent with the similarity map. More precisely, the indexing is an instrument how the mapping is to be chaotic. Moreover, trajectories move through different sets, and even various metric spaces, when the time increases, and each of the sets admits its own chaotic structure. This is why, we say that the modular chaos is in the focus of the paper or more precisely modular domain-structured chaos.

In the paper [4] the notion of domain-structured chaos is presented in most abstract form, free of topological properties for domains. This time, we intend to consider the chaos developed to the level, when two points are close, if they belong to the same set by indexing.

It is meaningful that the approach helps us to find a chaotic structure for continuous-time random processes, bounded as well as unbounded. We accept the phenomenon as chaos presence in the dynamics, since realizations of the corresponding discrete time random process with fixed discrete sequence of time are the same as trajectories of the deterministic modular similarity map introduced in the present research. One must say that the concepts of Poincaré chaos and unpredictable sequences are of strong importance for the investigation.

We hope that the concept will be developed further, by considering multidimensional indexing and infinitely dimensional dynamics. Additionally, the definitions can be considered for processes, which are stochastic only partially, in time, that is hybrid dynamics, through combinations of intervals, where a dynamics is deterministic and/or stochastic. The suggestions can be considered for construction of hierarchy of chaos such that each next level of the complex dynamics can be determined as union of previous ones.

In the basis of our investigation are the most effective methods, which have been developed for chaos investigation through similarity, self-similaruty and symbolic dynamics.

The research on similarity goes back to G. Leibniz, who introduced the notions of recursive self-similarity [5]. The idea of a self-similar set was first considered by Moran, 6]. He gave a mathematical definition of a geometric construction as a collection of sets satisfying specific conditions. Further, definitions of self-similarity and related problems were discussed in papers 77-17. Our research is based on a point-set structure in metric spaces and this is why we call it abstract self-similarity [18. It does not rely on any special functions, and the similarity map is naturally combined with the concept. The map can be considered as a generalization of the Bernoulli shift on symbolic spaces [19. We suppose that next extension of the present results can be obtained on the basis of papers [10 17].

\footnotetext{
*Corresponding Author, Tel.: +90 312210 5355, Fax: +90 312210 2972, E-mail: marat@metu.edu.tr
} 
Potential applications of the method naturally relate to ways of chaos research such as chaos control and synchronization 20. The theory of stochastic differential equations 21] can be enriched, if modular chaos investigation results are involved in the study by utilizing methods of replication of chaos and unpredictable dynamics [3,22,23.

\section{Modular domain-structured chaos}

Let a countable collection of metric spaces $\left(F_{j}, d_{j}\right), j=1,2, \ldots$, be given, with distances $d_{j}$. Assume that for each set $F_{j}$ the following presentation exists,

$$
\mathcal{F}^{j}=\left\{\mathcal{F}_{i_{1} i_{2} \ldots i_{n} \ldots}^{j}: i_{k}=1,2, \ldots, m, k\right\}, j=1,2, \ldots,
$$

where $m$ is a natural number not smaller than two and common for all these sets. It means that each element of the set $F_{j}$ is labeled through at least by one member of $\mathcal{F}^{j}$, and each element of set $\mathcal{F}^{j}$ presents a member from $F_{j}$. We assume that the uniqueness is not necessarily required for the relation. That is, if $\mathcal{F}_{i_{1} i_{2} \ldots i_{n} \ldots}^{j}$ and $\mathcal{F}_{j_{1} j_{2} \ldots j n, \ldots}^{j}$ present the same element $f \in \mathcal{F}_{j}$, it is not necessary that $i_{n}=j_{n}$ for all $n=1,2, \ldots$ Moreover, we determine functions $\delta_{j}\left(\mathcal{F}_{i_{1} i_{2} \ldots i_{n} \ldots}^{j}, \mathcal{F}_{j_{1} j_{2} \ldots j n, \ldots}^{j}\right)=d_{j}\left(f_{1}, f_{2}\right), j=1,2, \ldots$, if $\mathcal{F}_{i_{1} i_{2} \ldots i_{n} \ldots}^{j}$ and $\mathcal{F}_{j_{1} j_{2} \ldots j n, \ldots}^{j}$ correspond to elements $f_{1}$ and $f_{2}$ of the set $F_{j}$ respectively, such that $\delta_{j}\left(\mathcal{F}_{i_{1} i_{2} \ldots i_{n} \ldots}^{j}, \mathcal{F}_{j_{1} j_{2} \ldots j n, \ldots}^{j}\right)=0$ for different presentations of the same point in $F_{j}$.

The following sets are needed,

$$
\mathcal{F}_{i_{1} i_{2} \ldots i_{n}}^{j}=\bigcup_{j_{k}=1,2, \ldots, m} \mathcal{F}_{i_{1} i_{2} \ldots i_{n} j_{1} j_{2} \ldots}^{j},
$$

where indices $i_{1}, i_{2}, \ldots, i_{n}$, are fixed. It is clear that

$$
\mathcal{F}^{j} \supseteq \mathcal{F}_{i_{1}}^{j} \supseteq \mathcal{F}_{i_{1} i_{2}}^{j} \supseteq \ldots \supseteq \mathcal{F}_{i_{1} i_{2} \ldots i_{n}}^{j} \supseteq \mathcal{F}_{i_{1} i_{2} \ldots i_{n} i_{n+1}}^{j} \ldots, i_{k}=1,2, \ldots, m, k=1,2, \ldots
$$

That is, the sets form a nested sequence.

We will say that the diameter condition is valid for sets $\mathcal{F}_{i_{1} i_{2} \ldots i_{n}}^{j}, j=1, \ldots$, if

$$
\max _{i_{1} i_{2} \ldots i_{n}} \operatorname{diam}\left(\mathcal{F}_{i_{1} i_{2} \ldots i_{n}}^{j}\right) \rightarrow 0 \text { as } n \rightarrow \infty,
$$

where $\operatorname{diam}(A)=\sup \left\{\delta_{j}(\mathbf{x}, \mathbf{y}): \mathbf{x}, \mathbf{y} \in A\right\}$, for a set $A$ in $\mathcal{F}_{j}$.

Determine the function $\delta_{j}(A, B)=\inf \left\{\delta_{j}(\mathbf{x}, \mathbf{y}): \mathbf{x} \in A, \mathbf{y} \in B\right\}$, for two nonempty bounded sets $A$ and $B$ in $\mathcal{F}_{j}$. The set $\mathcal{F}^{j}, j=1,2, \ldots$, satisfies the separation condition of degree $n$, if there exist a positive number $\varepsilon_{0}^{j}$ and a natural number $n=n(j)$ such that for arbitrary indices $i_{1} i_{2} \ldots i_{n}$ one can find indices $j_{1} j_{2} \ldots j_{n}$ such that

$$
\delta_{j}\left(\mathcal{F}_{i_{1} i_{2} \ldots i_{n}}^{j}, \mathcal{F}_{j_{1} j_{2} \ldots j_{n}}^{j}\right) \geq \varepsilon_{0}^{j} .
$$

If the diameter and separation conditions are valid, then $\mathcal{F}^{j}$ is said to be a chaotic structure for $F_{j}, j=1,2, \ldots$, and a chaotic module for $F$.

We shall say that the union, $\mathcal{F}$, of all modules $\mathcal{F}_{j}, j=1,2, \ldots$, is a modular chaotic structure for the union $F$ of all sets $F_{j}, j=1,2, \ldots$, if the diameter condition is valid for all $j=1,2, \ldots$, and there exists a positive separation constant $\varepsilon_{0}=\inf _{j=1,2, \ldots} \varepsilon_{0}^{j}$.

Let us introduce the maps $\varphi_{j}: \mathcal{F}^{j} \rightarrow \mathcal{F}^{j+1}, j=1,2, \ldots$, such that

$$
\varphi_{j}\left(\mathcal{F}_{i_{1} i_{2} \ldots i_{n} \ldots}^{j}\right)=\mathcal{F}_{i_{2} i_{3} \ldots i_{n} \ldots}^{j+1} .
$$

In what follows, we refer to the map $\varphi$ on the set $\mathcal{F}$, which is specified by equations (5). That is, $\varphi\left(\mathcal{F}^{j}\right)=$ $\varphi_{j}\left(\mathcal{F}^{j}\right), j=1,2, \ldots$ Moreover, accept that $\delta\left(\mathcal{F}_{i_{1} i_{2} \ldots i_{n} \ldots}^{j}, \mathcal{F}_{j_{1} j_{2} \ldots j_{n} \ldots}^{j}\right)=\delta_{j}\left(\mathcal{F}_{i_{1} i_{2} \ldots i_{n} \ldots}^{j}, \mathcal{F}_{j_{1} j_{2} \ldots j_{n} \ldots}^{j}\right), j=1,2, \ldots$

Considering iterations of the map, one can verify that

$$
\left(\varphi_{j+n}\left(\ldots\left(\varphi_{j}\left(\mathcal{F}_{i_{1} i_{2} \ldots i_{n}}^{j}\right)\right) \ldots\right)=\varphi^{n}\left(\mathcal{F}_{i_{1} i_{2} \ldots i_{n}}^{j}\right)\right)=\mathcal{F}^{j+n+1},
$$

for arbitrary natural number $n$ and $i_{k}=1,2, \ldots, m, k=1,2, \ldots$. The relations (5) and (6) give us a reason to call $\varphi$ an affine similarity map, since the shifts for lower and upper indexes are common. One can call also the map modular similarity map. Moreover, the number $n$ is named the order of similarity. Analogously to the research in [18, we will call the set $\mathcal{F}$ modular abstract self-similar set . 
The set $\mathcal{F}^{j}$, for a fixed $j=1,2, \ldots$, is an abstract self-similar set [18. If $\mathcal{F}^{j}$ does not depend on $j$, the modular similarity map $\varphi$ is known as the similarity map [4, 24].

It is clear that there is a naturally determined map $\Phi: F \rightarrow F$, which is specified as $\Phi: F_{j} \rightarrow F_{j+1}, j=1,2, \ldots$, and values $\Phi(f)$ for a fixed $f \in F_{j}$ constitute the set $\left\{\phi\left(\mathcal{F}_{i_{1} i_{2} \ldots i_{n} \ldots}^{j}\right)\right\}$, with all labels of the point $f$. The map $\Phi$ does not satisfy the uniqueness condition. In what follows, we shall describe chaotic properties of the map $\Phi$ in terms of the dynamics of the map $\phi$.

We shall say that an element $\mathcal{F}_{i_{1} i_{2} \ldots i_{n} \ldots}^{j}$ is in $(k, m)$-neighborhood of a point $\mathcal{F}_{s_{1} s_{2} \ldots s_{n} \ldots}^{p}, j<p$, if $\mathcal{F}_{i_{k+1} i_{k+2} \ldots i_{k+m}}^{j+k}=$ $F_{s_{1} s_{2} \ldots s_{m}}^{p}$. In other words, $j+k=p, i_{k+1}=s_{1}, \ldots, i_{k+m}=s_{m}$.

Since the concepts are based on the Bernoulli shift [19], known for the symbolic dynamics, it is easily to see that the following chaotic properties are valid.

A point $\mathcal{F}_{i_{1} i_{2} i_{3} \ldots}^{j}, j=1,2, \ldots$, is periodic with period $p$, if its lower index consists of endless repetitions of a block of $p$ terms. The map $\varphi$ is said to be modular-periodic, since $\varphi^{k p}\left(\mathcal{F}_{i_{1} i_{2} \ldots i_{n} \ldots}^{j}\right)=\mathcal{F}_{i_{1} i_{2} \ldots i_{n} \ldots}^{j+k p}$ for any natural $k$, if the point $\mathcal{F}_{i_{1} i_{2} \ldots i_{n} \ldots}^{j} \in \mathcal{F}^{j}$ is $p$-periodic.

Similarly, the map is modular-dense or modular-transitive, since there exists the lower index $i_{1} i_{2} i_{3} \ldots$, such that for any sequence $j_{1} j_{2} j_{3} \ldots$ and natural $j$ and $l$ one can find positive integers $m$ and $k, j<k$, such that $\varphi^{m}\left(\mathcal{F}_{i_{1} i_{2} \ldots i_{n} \ldots}^{j}\right)=\mathcal{F}_{j_{1} j_{2} \ldots j_{n} \ldots}^{k}$, and $i_{s}=j_{s}, s=1, \ldots, l$. In other words, $\mathcal{F}_{i_{1} i_{2} \ldots i_{n} \ldots}^{j}$ is in $(m, l)-$ neighborhood of $\mathcal{F}_{j_{1} j_{2} \ldots j_{n} \ldots}^{k}$.

Finally, one can say that the periodic points are modular-dense in $\mathcal{F}$, since for any point $\mathcal{F}_{j_{1} j_{2} \ldots j_{n} \ldots}^{k}$ of the set and natural numbers $l$ and $m \leq k$ one can find a periodic point $\mathcal{F}_{i_{1} i_{2} \ldots i_{n} \ldots}^{m}$ such that it is in $(k-m, l)-$ neighborhood of $\mathcal{F}_{j_{1} j_{2} \ldots j_{n} \ldots}^{k}$.

It is clear that couples $\left(\mathcal{F}^{j}, \delta_{j}\right), j=1,2, \ldots$, are not, in general, metric spaces. Nevertheless, the map $\varphi$ is convenient to extend all attributes of Poincaré, Li-Yorke and Devaney chaos [1, 2, 22, for the dynamics, since the spaces $\left(F_{j}, d_{j}\right)$ are metric. We shall say that the map $\varphi$ is modular chaotic in the Devaney sense if it is dense with respect to modular-periodic trajectories, modular-transitive and modular-sensitive. The modular-sensitivity means, that there exists a positive number $\varepsilon_{0}$ such that for each element $\mathcal{F}_{i_{1} i_{2} \ldots i_{n} \ldots}^{j}$ and arbitrary positive $\kappa$ one can find an element $\mathcal{F}_{j_{1} j_{2} \ldots j_{n} \ldots}^{j}$ and a natural number $k$, which satisfy $\delta\left(\varphi^{k}\left(\mathcal{F}_{i_{1} i_{2} \ldots i_{n} \ldots}^{j}\right), \varphi^{k}\left(\mathcal{F}_{j_{1} j_{2} \ldots j_{n} \ldots}^{j}\right)>\varepsilon_{0}\right.$, despite $\delta\left(\mathcal{F}_{i_{1} i_{2} \ldots i_{n} \ldots}^{j}, \mathcal{F}_{j_{1} j_{2} \ldots j_{n} \ldots}^{j}\right)<\kappa$. Finally, the map $\Phi$ is modular chaotic in the sense of Devaney, if the corresponding $\operatorname{map} \varphi$ is modular chaotic in the same sense.

Theorem 1. If $\mathcal{F}$ is a modular chaotic structure, then the dynamics of $\Phi$ is modular chaotic in the sense of Devaney.

Proof. Modular-transitivity and density for modular-periodicity are valid, since of the definitions. For modularsensitivity, fix a point $\mathcal{F}_{i_{1} i_{2} \ldots}^{j} \in \mathcal{F}$ and an arbitrary positive number $\kappa$. Due to the diameter and separation conditions, there exist an integer $k$ and element $\mathcal{F}_{i_{1} i_{2} \ldots i_{k} j_{k+1} j_{k+2} \ldots}^{j}$ such that $0<\delta\left(\mathcal{F}_{i_{1} i_{2} \ldots i_{k} i_{k+1} \ldots}^{j}, \mathcal{F}_{i_{1} i_{2} \ldots i_{k} j_{k+1} j_{k+2} \ldots}^{j}\right)<\kappa$ and $\delta\left(\mathcal{F}_{i_{k+1} i_{k+2} \ldots i_{k+n}}^{j}, \mathcal{F}_{j_{k+1} j_{k+2} \ldots j_{k+n}}^{j}\right)>\varepsilon_{0}$. This proves the sensitivity.

In [3.25], Poisson stable motion is utilized to distinguish chaotic behavior from periodic motions in Devaney and Li-Yorke types. The dynamics is named Poincarè chaos. Let us provide the definition adapted for the dynamics of the present research. A point $\mathcal{F}_{i_{1} i_{2} \ldots}^{j} \in \mathcal{F}, j=1,2, \ldots$, is said to be the modular unpredictable point for the map $\varphi$, if there exist a positive number $\varepsilon_{0}$ and two unbounded sequences of natural numbers, $\kappa_{n}$ and $\zeta_{n}, n=1,2, \ldots$, such that for arbitrary natural number $l$ one can find sufficiently large $\kappa_{n}$ such that $\varphi^{\kappa_{n}}\left(\mathcal{F}_{i_{1} i_{2} \ldots}^{j}\right)=\mathcal{F}_{j_{1} j_{2} \ldots}^{j+\kappa_{n}}$, where $j_{s}=i_{s}, s=1, \ldots, l$, and $j_{\kappa_{n}+\zeta_{n}} \neq i_{\kappa_{n}+\zeta_{n}}$.

Following the definition of Poincaré chaos [3] we shall say that the dynamics of the map $\varphi$ is modular-chaotic in the sense of Poincaré if there is a modular-unpredictable point for each $j=1,2, \ldots$ Moreover, we call the unpredictable point itself an unpredictable orbit of the dynamics. It is natural to accept that the map $\Phi$ is modular chaotic in the sense of Poincaré, if the corresponding map $\varphi$ is modular chaotic in the sense of Poincaré.

Theorem 2. If $\mathcal{F}$ is a modular chaotic structure, then the map $\Phi$ possesses modular chaos in the sense of Poincarè.

The proof of the last theorem is based on the verification of Lemma 3.1 in [3], applied to the modular similarity map.

In addition to modular Devaney and Poincarè chaos, the chaos of the Li-Yorke type [2] can be defined. Additionally to the modular-periodic points of the dynamics, assume that there exists an uncountable scrambled set set of non-periodic points in $\mathcal{F}^{j}, j=1,2, \ldots$, such that

$$
(\alpha) \lim \sup _{k \rightarrow \infty} \delta\left(\varphi^{k}\left(\mathcal{F}_{i_{1} i_{2} \ldots}^{j}\right), \varphi^{k}\left(\mathcal{F}_{i_{j} j_{2} \ldots}^{j}\right)\right)>0
$$


and

$$
(\beta) \lim \inf _{k \rightarrow \infty} \delta\left(\varphi^{k}\left(\mathcal{F}_{i_{1} i_{2} \ldots}^{j}\right), \varphi^{k}\left(\mathcal{F}_{i_{j} j_{2} \ldots}^{j}\right)\right)=0,
$$

for each couple in the set, if $\delta\left(\mathcal{F}_{i_{1} i_{2} \ldots}^{j}, \mathcal{F}_{i_{j} j_{2} \ldots}^{j}\right) \neq 0$. And, finally, assume that for each point $\mathcal{F}_{i_{1} i_{2} \ldots}^{j}$ of the set and a periodic point $\mathcal{F}_{i_{j} j_{2} \ldots}^{j}$ it is true that $\lim \sup _{k \rightarrow \infty} \delta\left(\varphi^{k}\left(\mathcal{F}_{i_{1} i_{2} \ldots}^{j}\right), \varphi^{k}\left(\mathcal{F}_{i_{j} j_{2} \ldots}^{j}\right)\right)>0$. The conditions $(\alpha)$ and $(\beta)$ are called modular-proximality and modular-frequent separation properties for the modular Li-Yorke chaos, respectively.

We will say that for the sets $\mathcal{F}_{i_{1} i_{2} \ldots i_{n}}^{j}, j=1, \ldots$, the strong diameter condition is valid, if

$$
\sup _{j} \max _{i_{1} i_{2} \ldots i_{n}} \operatorname{diam}\left(\mathcal{F}_{i_{1} i_{2} \ldots i_{n}}^{j}\right) \rightarrow 0 \text { as } n \rightarrow \infty \text {. }
$$

We shall say that the union, $\mathcal{F}$, of all modules $\mathcal{F}_{j}, j=1,2, \ldots$, is a strong modular chaotic structure for the union $F$ of all sets $F_{j}, j=1,2, \ldots$, if the strong diameter condition is valid for all $j=1,2, \ldots$, and there exists a positive separation constant $\varepsilon_{0}=\inf _{j=1,2, \ldots} \varepsilon_{0}^{j}$. We shall call the map $\Phi$ modular chaotic in the sense of Li-Yorke, if the corresponding map $\varphi$ is modular chaotic in the sense of Li-Yorke.

Theorem 3. If $\mathcal{F}$ is a strong modular chaotic structure, then the map $\Phi$ is modular chaotic in the Li-Yorke sense.

The proof of the assertion is similar to that of Theorem 6.35 in [26 for the shift map defined in the space of symbolic sequences.

One can easily see that, in the case when $\left(F_{j}, d_{j}\right)$ does not depend on $j$, we obtain domain-structured chaos, which was introduced in [4. That is, modular chaos can be considered as generalization of domain-structured chaos. The modular chaos relates to domain structured chaos similarly as non-autonomous dynamics does to autonomous one.

The analysis of ingredients for the three types of chaos implies that the most weak, and, consequently, most flexible properties are required for Poincaré modular chaos, since there distance evaluations are not request. Nevertheless, we are confident that this must not be considered as a deficiency. The description still can provide circumstances, sufficient for irregularity, which can be useful for future applications.

\section{Chaotic random processes}

Let us describe the type of the stochastic processes [27] that are in the focus of this paper. Consider a continuous time or discrete time random process with continuous or discrete state space as a family of random variables $\mathbf{X}(t)$ indexed by the parameter $t$ with the range $\mathcal{I}$, which is either an interval of the real line or an infinite set of integers bounded from below. Realizations of the dynamics are not necessarily continuous if the time is continuous and they must not be bounded functions for both sorts of time. Denote by $S=\bigcup_{t \in \mathcal{I}} S^{t}$ the state space of the process, and consider it with a distance $d$. We assume positive probability for all members of the state space, and for each fix $t$ one of the members of the state space must necessarily happen.

Suppose that for each fixed $t \in \mathcal{I}$ there exists a presentation,

$$
\mathcal{S}^{t}=\left\{\mathcal{S}_{i_{1} i_{2} \ldots i_{n} \ldots}^{t}: i_{k}=1,2, \ldots, m, k=1,2, \ldots\right\},
$$

where $m$ is a fixed natural number, common for all $t \in \mathcal{I}$, larger or equal than two, such that each element of the set $S^{t}$ is presented as a member of the set $\mathcal{S}^{t}$. The presentation is in the sense of the last section, and corresponding to the distance $d$ function $\delta$ is common for all $t \in \mathcal{I}$.

Consider all possible infinite strictly increasing sequences $t_{\alpha}=\left\{t_{i}^{\alpha}\right\}, i=1,2, \ldots$, in $\mathcal{I}$, with $\mathcal{A}$, the set of all indexes $\alpha$.

We shall say that the random process $\mathbf{X}(t)$ is (strongly) modular chaotic, if $\mathcal{S}^{\alpha}=\bigcup_{i} \mathcal{S}_{i}^{\alpha}$ is a (strong) modular chaotic structure for $S^{\alpha}=\bigcup_{i} S^{t_{i}^{\alpha}}$ with each $\alpha \in \mathcal{A}$, and there exists a number $\varepsilon_{0}$, which is larger than all separation constants $\varepsilon_{0}^{\alpha}, \alpha \in \mathcal{A}$.

The choice of modular chaos for the random dynamics description is approved, since the set of all realizations for dynamics $\mathbf{X}\left(t_{\alpha}\right)$ with fixed $\alpha \in \mathcal{A}$ coincides with the set of all trajectories of the affine similarity map $\varphi$ on the set $\mathcal{S}^{\alpha}$, and the dynamics of the affine map is similar to the symbolic dynamics. To argument this, let us turn the discussion to the lower indexes analysis. Consider the space $\Sigma_{m}=\left\{\left(i_{1} i_{2} \ldots\right) \mid i_{k}=1,2, \ldots, m\right\}$ of infinite sequences on finite number of symbols with the metric

$$
d(s, t)=\sum_{k=1}^{\infty} \frac{\left|i_{k}-j_{k}\right|}{2^{k-1}},
$$


where $\left(i_{1} i_{2} \ldots\right)$, and $\left(j_{1} j_{2} \ldots\right)$ are elements of $\Sigma_{m}$. The Bernoulli shift $\sigma: \Sigma_{m} \rightarrow \Sigma_{m}$ is defined as $\sigma\left(s_{0} s_{1} s_{2} \ldots\right)=$ $\left(s_{1} s_{2} s_{3} \ldots\right)$. The map $\sigma$ is continuous and $\Sigma_{r}$ is a compact metric space [1,19]. According to the result in [3], the symbolic dynamics admits an unpredictable point, $i^{*}$, a sequence from the set [25. It is important for us that $\Sigma_{m}$ is a closure for the unpredictable orbit. The peculiarity of the metric implies that each arc of any sequence in the space coincides with some arc of the unpredictable sequence with a shift precision. In other words, since of finite number of indexes involved in the construction, one have the complete coincidence with a part of the unpredictable orbit. Moreover, the coincidence does not last forever, since of the unpredictability. This is why, each iteration of the Bernoulli shift $\sigma$ results what is done by the Bernoulli trial and, similarly, by $\mathbf{X}\left(t_{\alpha}\right)$. This is why, we accept that the random dynamics is modular chaotic.

It is clear that for the discrete time random process, it is sufficient to consider only the sequence related to the set of indexes $\mathcal{I}$ itself. We do not exclude that the method of modular chaos may be of use even if the modular chaotic structure exists not for all $\alpha$ from $\mathcal{A}$, but for some of them.

On the basis of the last discussion one can say that stochastic processes are more "saturated with irregularity", than deterministic chaos. Nevertheless, we have the strong confidence that through the developed results one can make the difference between the two concepts less, if not diminish at all. Next, we will provide three examples of random processes, which are modular chaotic.

Example 1. Consider several scalar real-valued functions $f_{j}(t), j=1, \ldots, m$, defined on a time interval, $\mathcal{I}$, of real numbers. Assume that the ranges of the functions are disjoint and minimal distance between elements of distinct ranges is larger than a positive number $\varepsilon_{0}$. Define the random process $\boldsymbol{X}(t)$ such that for each fixed $t \in \mathcal{I}$, it admits value of one of the functions $f_{j}$ with positive probability $p_{j}, j=1, \ldots, m$. The probabilities do not depend on $t$, and the sum of all probabilities is equal to the unit if $t$ is fixed.

We have that the state space is $S=\bigcup_{t \in \mathcal{I}} S^{t}$, where $S^{t}=\left\{f_{j}(t) \mid j=1, \ldots, m\right\}$. Let us construct the presentation

$$
\mathcal{S}^{t}=\left\{\mathcal{S}_{i_{1} i_{2} \ldots i_{n} \ldots}^{t}: i_{k}=1,2, \ldots, m, k=1,2, \ldots\right\},
$$

where $\mathcal{S}_{i_{1} i_{2} \ldots i_{n} \ldots}^{t}=f_{i_{1}}(t), i_{j}=1,2, \ldots, m, j=2, \ldots$ One can check that the union, $\mathcal{S}$, of sets $\mathcal{S}^{t}, t \in \mathcal{I}$, is a modular chaotic structure for the set $S$, and consequently the random process is modular chaotic.

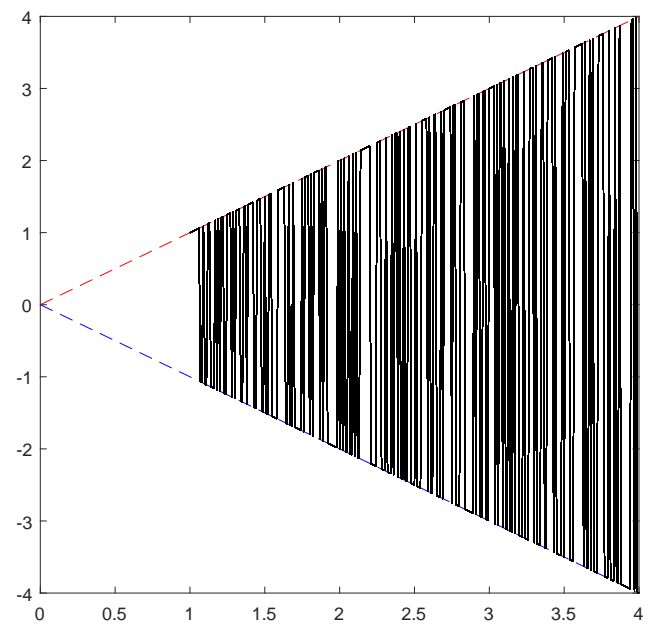

Figure 1: The graph of the function $\varphi$ in interval $[1,4]$. The red dashed line is the graph of the identity function $t$ and the blue dashed line is the graph of function $-t$.

Example 2. (numerical simulation) Let us consider the random process $\boldsymbol{X}(t)$, which is equal to $t$ or $-t$ for each fixed $t \in[1,4]$, with probability $1 / 2$. It is clear that the dynamics is a continuous-time random process with continuous state space. Fix the parameter value $t$ and obtain that $S^{t}=\{t,-t\}$ consists of two elements, and the distance between the two points is equal $|t-(-t)|=2 t \geq \varepsilon_{0}=2$. Let us, construct a chaotic structure for the set $S^{t}$. Denote $S_{i_{1} i_{2} \ldots}^{t}=t$ with $i_{1}=1$, and $i_{j}$ is equal to 1 or 2 for all $j \geq 2$. Similarly, $S_{i_{1} i_{2} \ldots}^{t}=-t$ with $i_{1}=2$ and $i_{j}$ is equal to 1 or 2 for all $j \geq 2$. According to discussion in the last example the random process is modular chaotic. To illustrate 
the dynamics, we consider the sequence $t_{i}=i / 100, i=100,2, \ldots, 400$, randomly determine values $\boldsymbol{X}\left(t_{i}\right)$ and draw the graph of the piece-wise constant function $\varphi$ equal to $\boldsymbol{X}\left(t_{i}\right)$ on the interval $[i / 100,(i+1) / 100), i=100, \ldots, 399$. The graph is seen in Figure 1 and it approximates one of realizations of the random dynamics.

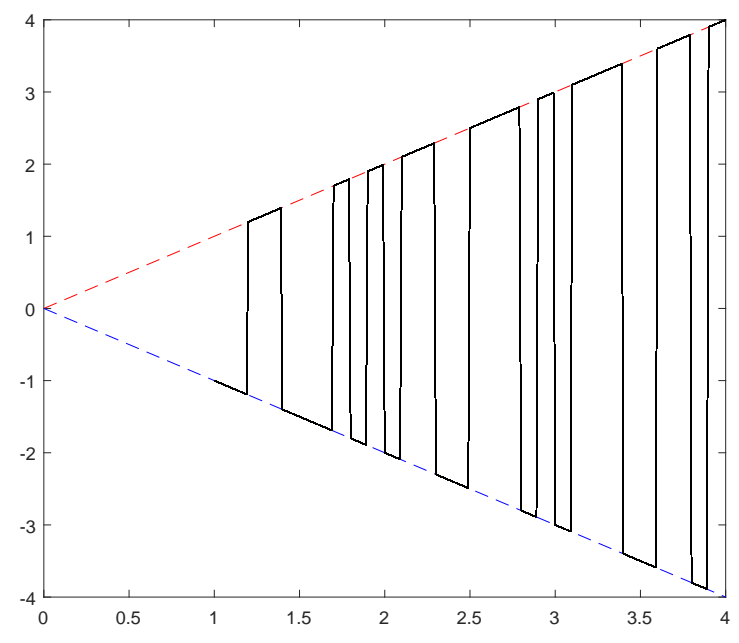

Figure 2: The graph of the function $\psi$ in interval $[1,4]$. The red dashed line is the graph of the identity function $t$ and the blue dashed line is the graph of function $-t$.

Example 3. (discrete time dynamics) Let us determine functions $f_{i}(t)=t$ and $g_{i}(t)=-t$ on the interval $[i / 10,(i+$ 1)/10) for each $i=10,11, \ldots$ Construct the metric space of continuous functions, $S^{i}=\left\{f_{i}(t), g_{i}(t)\right\}, t \in[i / 10,(i+$ $1) / 10)$, for each $i=10,11, \ldots$, with the distance $d\left(f_{i}(t), g_{i}(t)\right)=\max _{t}|t-(-t)|$. Introduce the discrete time random process $\boldsymbol{X}(i), i=10,11, \ldots$, which is equal to the function $f_{i}(t)$ or $g_{i}(t)$ with probability $1 / 2$ on the interval $[i / 10,(i+$ $1) / 10)$. One can easily see that the random dynamics is modular chaotic, since one can construct appropriate modular chaotic structure. The graph of a realization, $\psi$, on the interval $[1,4]$ is seen in Figure Q

\section{Conclusion}

We have pursued three goals in the present research. The first one is to reveal deterministic chaos signs in random phenomena, and the second, to maximize the presence. New definitions of chaos to fulfill the two tasks have been developed. All the three are considered in this research for random processes with discrete and continuous time. The application of the modular chaos concept to random dynamics analysis is sensible, since the set of all realizations of a random dynamics coincides with the set of all trajectories of the chaos. To approve the phenomenon, the domain structured chaos notion, which has been considered in previous our papers, is extended. The new type of chaos has been developed such that the notions of transitivity and density of periodic orbits are free of metrical or topological features, and are based on the comprehension that two points are close, if they belong to the same set by indexing. Moreover, trajectories move among different sets, when the time increases, and each of the sets admits its own chaotic structure. It is important that the concept helped us to find a chaotic structure for continuous-time random processes, bounded as well as unbounded. We accept the phenomenon as a sign of deterministic chaos in the dynamics, since realizations of the corresponding discrete time random process are the same as trajectories of the deterministic modular similarity map introduced in the present research. The concept can be considered for processes, which are stochastic only partially, in time, that is hybrid dynamics, through combinations of intervals, where a dynamics is deterministic or stochastic. Hopefully, this all may generates new approaches inside of the stochastic dynamics theory [21] as well as for non-stationary processes and for Markov chains. Our research results may help for development of chaos in non-equilibrium processes [28. The methods of chaotic dynamics, e.g. synchronization and control [20, can be extended to dynamics with probability. Moreover, one can expect that results for symbolic dynamics 29] considered in the course of the present research can give new effects for entropy [5],30, harmonic analysis, discrete mathematics, probability, and operator algebras [7. 


\section{References}

[1] Devaney, R. L. 1987, An Introduction to Chaotic Dynamical Systems. Addison-Wesley, Menlo Park.

[2] Li, T. Y. and Yorke, J. A. 1975, Period Three Implies Chaos. Amer. Math. Monthly 82 985-992.

[3] Akhmet, M. and Fen, M. O. 2016, Poincar chaos and unpredictable functions. Commun. Nonlinear Sci. Numer. Simulat. 48 85-94.

[4] M. Akhmet, 2019, Domain-structured chaos in discrete random processes, Arxiv e-prints, arXiv:1912.10478 (submitted).

[5] Zmeskal, O., Dzik, P. and Vesely, M. 2013, Entropy of fractal systems. Comput. Math. Appl. 66135146.

[6] Moran, P. A. P. 1946, Additive functions of intervals and Hausdorff measure. Proc. Cambridge Philos. Soc. 42 $15-23$.

[7] Jorgensen, Palle E. T. 2006, Analysis and Probability: Wavelets, Signals, Fractals. Graduate Texts in Mathematics, vol. 234, Springer, New York.

[8] Stella, S. 1992, On Hausdorff Dimension of Recurrent Net Fractals. Proc. Amer. Math. Soc. 116 389-400.

[9] Pesin, Y. and Weiss, H. 1996, On the Dimension of Deterministic and Random Cantor-like Sets, Symbolic Dynamics, and the Eckmann-Ruelle Conjecture. Comm. Math. Phys. 182 105-153.

[10] Hutchinson J. 1981, Fractals and self-similarity. Indiana Univ. J. Math. 30713747.

[11] Hata, M. 1985, On the structure of self-similar sets. Japan J. Appl. Math. 2 381-414.

[12] Edgar, G. A. 1990, Measure, Topology, and Fractal Geometry. Springer-Verlag, New York.

[13] Spear, D. W. 1992, Measure and self-similarity, Adv. Math. 91 143-157.

[14] Bandt, C. and Graf, S. 1992, Self-similar sets 7. A characterization of self-similar fractals with positive Hausdorff measure. Proc. Amer. Math. Soc. 1149951001.

[15] Falconer, K. J. 1995, Sub-self-similar sets, Trans. Amer. Math. Soc. 347(4) 31213129.

[16] Lau, K. S., Ngai, S. M. and Rao, H. 2001, Iterated function systems with overlaps and the self-similar measures. J. London Math. Soc. 6399115.

[17] Ngai, S. M. and Wang, Y. 2001, Hausdorff dimension of overlapping self-similar sets. J. London Math. Soc. 63(2) 655672.

[18] M. Akhmet, E. M. Alejaily, 2020, Abstract similarity, chaos and fractals, Discrete and Continuous Dynamical Systems, Ser. B. ( accepted)

[19] S. Wiggins, 1988, Global Bifurcation and Chaos: Analytical Methods, Springer-Verlag, New York, Berlin.

[20] J.M. Gonzáles-Miranda, 2004, Synchronization and control of chaos, Imperial College Press, London.

[21] B.K. Oksendal, 2003, Stochastic Differential Equations: An Introduction with Applications, Springer, Berlin.

[22] M. Akhmet, M.O. Fen and E. M. Alejaily, 2020, Dynamics with chaos and fractals, Springer.

[23] M. Akhmet, M.O. Fen, 2016, Replication of chaos in neural networks, economics and physics. Nonlinear Physical Science, Springer, Higher Education Press, Beijing, Heidelberg.

[24] M. Akhmet, E. M. Alejaily, 2019, Domain-Structured Chaos in a Hopfield Neural Network, Int. J. Bifurc. Chaos, 29 (14), article . Article number1950205.

[25] Akhmet, M. and Fen, M. O. 2016, Unpredictable points and chaos. Commun. Nonlinear Sci. Numer. Simulat. $401-5$.

[26] G. Chen, Y. Huang, 2011, Chaotic Maps: Dynamics, Fractals and Rapid Fluctuations, Synthesis Lectures on Mathematics and Statistics, Morgan and Claypool Publishers, Texas. 
[27] J. L. Doob, 1953, Stochastic processes, Wiley, New York.

[28] G. Nicolis, I. Prigogine, 1989, Exploring complexity: An Introduction, Freeman and company, New York.

[29] S. Williams (editor), 2004, Symbolic Dynamics and its Applications. Proc. Symp. Appl. Math. 60.

[30] R. Adler, A. Konheim, and M. McAndrew, 1965, Topological entropy.Trans. Amer. Math. Soc.,114 309-319. 